\title{
The gene encoding the iron regulatory peptide hepcidin is regulated by anemia, hypoxia, and inflammation
}

\author{
Gaël Nicolas, ${ }^{1}$ Caroline Chauvet, ${ }^{2}$ Lydie Viatte, ${ }^{1}$ Jean Louis Danan, ${ }^{2}$ Xavier Bigard, ${ }^{3}$ \\ Isabelle Devaux,${ }^{4}$ Carole Beaumont, ${ }^{4}$ Axel Kahn,${ }^{1}$ and Sophie Vaulont ${ }^{1}$ \\ ${ }^{1}$ Département de Génétique, Développement et Pathologie Moléculaire, Institut Cochin, INSERM, Centre National de la \\ Recherche Scientifique, et Université René Descartes, Faculté de Médecine Cochin-Port Royal, Paris, France \\ ${ }^{2}$ Centre de Recherche sur l'Endocrinologie Moléculaire et le Développement, Centre National de la Recherche Scientifique \\ UPR9078, Meudon-Bellevue, France \\ ${ }^{3}$ Unité de Bioénergétique et Environnement, Centre de Recherches du Service de Santé des Armées, La Tronche Cedex, France \\ ${ }^{4}$ INSERM 409, Faculté de Médecine Xavier Bichat, Paris, France
}

\begin{abstract}
The present study was aimed at determining whether hepcidin, a recently identified peptide involved in iron metabolism, plays a role in conditions associated with both iron overload and iron deficiency. Hepcidin mRNA levels were assessed in two models of anemia, acute hemolysis provoked by phenylhydrazine and bleeding provoked by repeated phlebotomies. Hepcidin response to hypoxia was also studied, both ex vivo, in human hepatoma cells, and in vivo. Anemia and hypoxia were associated with a dramatic decrease in liver hepcidin gene expression, which may account for the increase in iron release from reticuloendothelial cells and increase in iron absorption frequently observed in these situations. A single injection of turpentine for 16 hours induced a sixfold increase in liver hepcidin mRNA levels and a twofold decrease in serum iron. The hyposideremic effect of turpentine was completely blunted in hepcidin-deficient mice, revealing hepcidin participation in anemia of inflammatory states. These modifications of hepcidin gene expression further suggest a key role for hepcidin in iron homeostasis under various pathophysiological conditions, which may support the pharmaceutical use of hepcidin agonists and antagonists in various iron homeostasis disorders.
\end{abstract}

J. Clin. Invest. 110:1037-1044 (2002). doi:10.1172/JCI200215686.

\section{Introduction}

Iron is an essential element required for growth and survival. This metal serves as a cofactor for many heme and nonheme iron proteins. However, excess free iron is toxic for the cell. Because mammals lack a regulated pathway for iron excretion, iron balance is maintained by the tight regulation of iron absorption from the intestine. The intestinal iron absorption is modulated in response to the level of body iron stores and by the amount of iron needed for erythropoiesis. This regulation is thought to operate through two regulators, namely, the store regulator and the erythroid regulator (for review, see ref. 1). When the amount of iron in the body stores decreases, the store regulator increases iron uptake until the reserves are replete. When iron stores are increased, it reduces intestinal iron absorption, thus preventing iron overload. Although the store regulator is capable of

Received for publication April 15, 2002, and accepted in revised form July 25, 2002.

Address correspondence to: Sophie Vaulont, Département de Génétique, Développement et Pathologie Moléculaire, Institut Cochin, Faculté de Médecine Cochin-Port Royal, 24 Rue du Faubourg Saint Jacques, 75014 Paris, France.

Phone: (33)1 44412408; Fax: (33)1 44412421;

E-mailvaulont@cochin.inserm.fr.

Conflict of interest: No conflict of interest has been declared. Nonstandard abbreviations used: $\mathrm{PHZ}$, phenylhydrazine; red blood cell (RBC); hemoglobin (HGB); hematocrit (HCT); mean cell volume (MCV). changing iron absorption to a relatively modest extent, its function is critical for normal iron homeostasis. The store regulator probably acts at the level of duodenal crypt cells, which become programmed in response to body store information, to influence the absorptive capacity of the daughter enterocytes.

As for the erythroid regulator, it is believed to transmit to the enterocyte the erythroid demand of the organism, iron absorption being upregulated as marrow iron requirement for erythropoiesis increases. This regulator operates over a broader range than the store regulator and probably increases intestinal iron absorption independently of the store regulator. However, an increase in erythropoiesis alone is not enough to increase iron absorption. Rather, it is the imbalance between the rate of erythropoiesis of the marrow and its iron supply that is thought to induce iron absorption. Anemia-associated hypoxia could be one of the mechanisms by which the erythroid cue is transmitted to the duodenum. Indeed, hypoxia by itself is known to increase intestinal iron absorption (2).

Both the store regulator and the erythroid regulator are believed to be soluble components of the plasma able to communicate between the sites of iron utilization and mobilization and the intestinal cells. So far, the signaling pathway and molecular components involved in the regulation of iron absorption through these two regulators have remained elusive. Recently, we proposed the circulating peptide hepcidin as a new 
candidate of the long-postulated store regulator. Hepcidin is a disulfide-bonded peptide exhibiting antimicrobial activity $(3,4)$. It has been purified from human blood ultrafiltrate and from urine. It is synthesized predominantly in the liver as an 84-amino acid precursor that is subsequently processed and secreted as a 25-amino acid peptide form (3-5). Several lines of evidence strongly suggest that this peptide plays a major role in the control of iron homeostasis. In mice experimentally induced iron loading in the liver was found to be associated with increased hepcidin gene expression (5). We demonstrated that a complete lack of hepcidin in mice leads to progressive iron accumulation resembling the iron overload of human hemochromatosis, with excess iron in hepatocytes and iron sparing of the reticuloendothelial cells (6). Finally, we demonstrated recently that transgenic animals overexpressing hepcidin in the liver have decreased body iron levels and presented at birth a severe microcytic hypochromic anemia (7). Taken together, these results highlighted the role of hepcidin as an iron regulator whose induction results in a decrease in both dietary iron absorption and transplacental iron transport.

In this article we further address the physiological role of hepcidin in vivo. We first ask whether hepcidin could play a role as an erythroid regulator, contributing to the modulation of iron absorption by the intestine and iron release by the macrophages in the situation of anemia with normal or increased iron stores. To this end, we investigated the pathophysiological response of hepcidin in situations associated with increased iron absorption, such as bleeding, phenylhydrazine-induced anemia, and hypoxia. Secondly, we ask whether hepcidin plays a role during the inflammatory states that are associated with decreased intestinal iron absorption and increased sequestration of iron in reticuloendothelial cells. This question was assessed by studying inflammation induced by turpentine in wildtype mice as well as in hepcidin-deficient mice.

\section{Methods}

Animals. All animals used in the experiments were cared for in accordance with criteria outlined in the European Convention for the Protection of Laboratory Animals. Animals were maintained in a temperature- and lightcontrolled environment and were given free access to tap water and food (standard laboratory mouse chow, AO3; Usine d'Alimentation Rationnelle, Epinay-sur-Orge, France). All mice, except the hepcidin-deficient mice (6), had a C57BL/6 genetic background. The control mice throughout the study were subjected to experimental manipulations similar to those of the treated mice.

Animal treatment. Chronic inflammation (abscess) was produced by subcutaneous injection of turpentine oil $(0.1 \mathrm{ml} / 20 \mu \mathrm{g}$ of body weight; Sigma-Aldrich, St. Louis, Missouri, USA) into the intrascapular fat pad under anesthesia at weekly intervals for 2 weeks (three injections). Control mice were similarly injected with an equivalent volume of sterile saline solution
$(0.15 \mathrm{M} \mathrm{NaCl})$. Acute-phase inflammation was produced with a single injection of turpentine oil (0.1 $\mathrm{ml} / 20$ g of body weight).

Phenylhydrazine (PHZ; Sigma-Aldrich) diluted in saline-buffered solution ( $40 \mathrm{mg} / \mathrm{kg}$ body weight, daily) was injected intraperitoneally on four consecutive days. Control mice were similarly injected with an equivalent volume of sterile saline solution $(0.15 \mathrm{M} \mathrm{NaCl})$. Mice were sacrificed the following day.

To induce anemia through phlebotomy, $0.5 \mathrm{ml}$ of blood was extracted by retro-orbital puncture from anesthetized mice. The procedure was repeated 16 hours later, and a third bleeding was performed 8 hours later. Animals were sacrificed 21 hours after the last phlebotomy. Control mice were subjected to sham phlebotomy.

For iron-dextran overload, two subcutaneous injections were performed 1 month apart, at the dose of $1 \mathrm{~g}$ of iron $/ \mathrm{kg}$ of body weight. Animals were sacrificed 1 month after the last injection. Some of these irondextran-treated animals were also treated with $\mathrm{PHZ}$ as mentioned above.

Hematological analysis of mice. Blood was obtained by retro-orbital phlebotomy before sacrifice of mice and collected in heparinized tubes (Capiject T-MLH; Terumo Medical Corp., Elkton, Maryland, USA). Blood cell counts and erythrocyte parameters were determined using a MaxM Coulter (Beckman Coulter Company, Hialeah, Florida, USA) automatic analyzer.

Cell culture and experimental hypoxia. Human hepatoma HepG2 cells (HB 8065; American Type Culture Collection, Rockville, Maryland, USA) were cultured as monolayers at $37^{\circ} \mathrm{C}$ in a humidified $95 \%$ air $5 \% \mathrm{CO}_{2}$ incubator. The culture medium used was a 1:1 mixture of DMEM and Ham-F12 media with Glutamax-I, supplemented with $10 \% \mathrm{FBS}, 100 \mu \mathrm{g} / \mathrm{ml}$ gentamicin, and 2.5 $\mu \mathrm{g} / \mathrm{ml}$ Fungizone (all from Invitrogen Life Technologies, Paisley, United Kingdom). HepG2 cells were made hypoxic by placing them in stainless steel hypoxia chambers as described (8). The chambers were evacuated until the desired oxygen partial pressure, deduced by measuring the pressure with an electronic pressure sensor (SMC digital pressure sensor; SMC Corporation, Tokyo, Japan) was reached. The chambers were then refilled with a gas mixture containing $5 \% \mathrm{CO}_{2}$ and $95 \% \mathrm{~N}_{2}$. Final oxygen concentrations of $10 \%, 2 \%$, or $0.1 \%$ were used. The chambers were then placed in an incubator at $37^{\circ} \mathrm{C}$ for 24 hours. Cells were still viable under these conditions (trypan blue exclusion, 98\% viability after 24 hours at $0.1 \% \mathrm{O}_{2}$ ).

In vivo hypoxia. Mice (C57BL/6 males aged 2 months) were housed in a hypobaric chamber and exposed to a reduced barometric pressure until the equivalent altitude of 5,500 $\mathrm{m}$ was reached within 6 hours (barometric pressure $=370 \mathrm{mmHg} ; \mathrm{PiO}_{2}=79 \mathrm{mmHg}$ ) (9). In the hypobaric chamber, mice were maintained at an ambient temperature of $22^{\circ} \pm 2^{\circ} \mathrm{C}$ with a 12 -hour dark/12hour light cycle and had free access to food and water. The chamber was opened daily to measure food consumption and refill water dispensers. 
RT-PCR and Northern blotting. The murine hepc1 transcripts were reverse-transcribed and amplified as described previously (6). Northern blot analysis was performed as described (6). Briefly, $20 \mu \mathrm{g}$ of RNA from each source was denatured in formaldehydecontaining buffer and electrophoresed in $1 \%$ agarose $/ 2.2 \mathrm{M}$ formaldehyde gels. After electrophoresis, RNA was transferred to a nylon membrane (GeneScreen Plus; Dupont-NEN, Boston, Massachusetts, USA) in $20 \times$ SSC buffer. The murine hepcidin cDNA probe, pT-Adv/hepc1, and the human VEGF probe, pcDNA-VEGF, were as described (see refs. 6, 10, respectively). The human hepc probe was made by RT-PCR from human liver RNA using the following primers: hHEPCF, $5^{\prime}$-GCACTGAGCTCCCAGATCTG-3', and hHEPCR, 5'-CTACGTCTTGCAGCACATCCC-3' (fragment size, $240 \mathrm{bp}$ ). Each blot was probed simultaneously with ribosomal $18 \mathrm{~S}$ cDNA to check for the integrity and the amount of loaded RNAs.

Iron measurements and histology. Quantification of iron level was performed as previously described by Torrance and Bothwell (11) on serum or liver using an IL test (Instrumentation Laboratory, Lexington, Massachusetts, USA).

\section{Results}

Hepcidin gene expression is decreased after experimentally induced anemia. To gain insight into the physiological response of hepcidin to experimentally induced acute anemia in mice, two models were selected, bleeding and PHZ treatment. In both cases, blood was collected to assess the parameters of the anemia, and hepcidin mRNA content in the liver was assessed by Northern blot analysis. Whereas there is only one copy of the hepcidin gene in the human genome, two highly related copies, bepc1 and hepc2, have been reported in the mouse. So far, there is no data assessing differential functional roles for bepc1 and hepc2. However, the hepc1 sequence is the most highly related to the human one, and we recently provided experimental data supporting a functional role for hepc1 in vivo (7). Therefore, the specific amount of hepc1 transcripts was also assessed by RT-PCR analysis.

For the phlebotomy protocol, a total of $1.5 \mathrm{ml}$ of blood was extracted by retro-orbital puncture (in three lots) over 24 hours. The experiment was performed 21 hours after the last bleeding. Blood parameters are presented in Figure 1a. Red blood cells (RBCs), hemoglobin (HGB), and hematocrit (HCT) values were reduced by approximately $70 \%$ in the phlebotomized mice, while the mean cell volume (MCV) was poorly affected. The Northern blot analysis revealed a $80 \%$ decrease in hepcidin mRNA in the liver of phlebotomized mice $(P<0.001$; Figure 1, b and c). RT-PCR assay showed a similar decrease for hepc1 transcripts (Figure 1d).

In the second model, anemia was induced by PHZ treatment. $\mathrm{PHZ}$ is a strong oxidant agent causing severe hemolytic anemia, which triggers vigorous erythropoiesis. Compared with saline-injected control animals, mice treated with $\mathrm{PHZ} 4$ days before the experiment showed induced reticulocytosis along with splenomegaly (not shown). The RBC and $\mathrm{HC}$ values were severely decreased ( $66 \%$ and $58 \%$ vs. control, respectively), while the MCV was increased (Figure 2a), consistent with a young cell population as a result of increased erythropoiesis. As shown in Figure 2b, Northern blot analysis indicated that hepcidin gene expression was strongly decreased after PHZ treatment (threefold reduction compared with salineinjected control, $P<0.0001$; Figure $2 \mathrm{c}$ ). RT-PCR analysis (Figure 2d) demonstrated that this diminution occurred for the bepc1 gene.
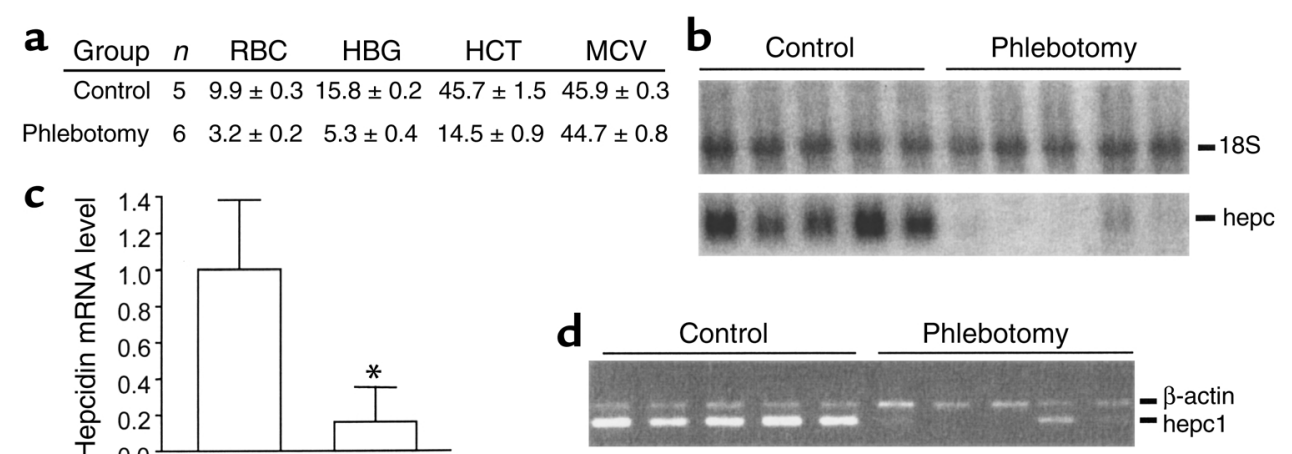

\section{Figure 1}

Hematological indices and hepcidin mRNA levels in phlebotomized mice. (a) Hematological parameters: RBC $\left(10^{6} / \mathrm{ml}\right), \mathrm{HGB}$ concentration (g/dl), HCT (\%), and MCV (femtoliter [fl]). Results are expressed as mean \pm SD for $n$ animals. (b) Hepcidin mRNA content in liver of control and phlebotomized mice as determined by Northern blot analysis. Twenty micrograms of total liver RNAs were electrophoresed, blotted, and hybridized with both hepcidin and 18S-labeled probes (as described in Methods). Autoradiography of a typical experiment. (c) Relative changes in hepcidin mRNA level (18S normalized, arbitrary units) as calculated using a STORM850 Phospholmager and ImageQuant 5.0. Results are expressed as mean \pm SD for $n$ animals, and statistical analysis was performed using the Student $t$ test (unpaired, twotailed). ${ }^{*} P<0.001$. (d) Level of specific hepc1 transcripts in the liver as measured by RT-PCR (as described in Methods). Following PCR, the amplified products (171 bp for hepc1 and $250 \mathrm{bp}$ for $\beta$-actin) were separated by electrophoresis on $1.5 \%$ agarose gel. 


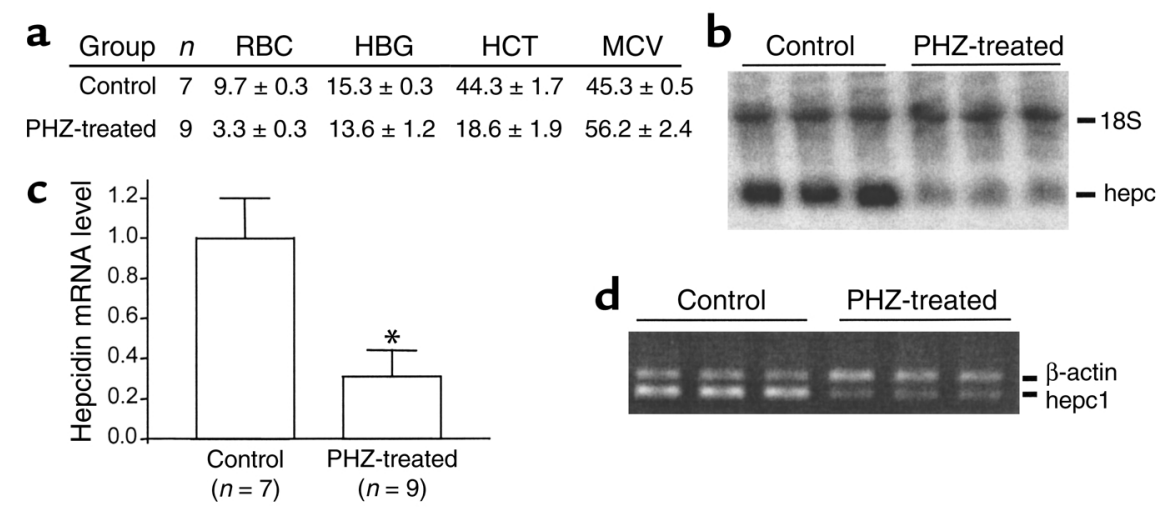

Figure 2

Hematological indices and hepcidin mRNA levels in PHZ-treated mice. (a) Hematological parameters: RBC $\left(10^{6} / \mathrm{ml}\right)$, HGB concentration $(\mathrm{g} / \mathrm{dl}), \mathrm{HCT}(\%)$, and $\mathrm{MCV}(\mathrm{fl})$. Results are expressed as mean \pm SD for $n$ animals. (b) Hepcidin mRNA content in liver of control and PHZ-treated mice as determined by Northern blot analysis. Twenty micrograms of total liver RNAs were electrophoresed, blotted, and hybridized with both hepcidin and 18S-labeled probes (as described in Methods). Autoradiography of a typical experiment. (c) Relative changes in hepcidin mRNA level (18S normalized, arbitrary units) as calculated using a STORM850 Phospholmager and ImageQuant 5.0. Results are expressed as mean \pm SD for $n$ animals, and statistical analysis was performed using the Student $t$ test (unpaired, two-tailed). ${ }^{*} P<0.0001$. (d) Level of specific hepc1 transcripts in the liver as measured by RT-PCR (as described in Methods). Following PCR, the amplified products ( $171 \mathrm{bp}$ for hepc 1 and $250 \mathrm{bp}$ for $\beta$-actin) were separated by electrophoresis on $1.5 \%$ agarose gel.

Because the hepcidin mRNA level was reported to be upregulated by iron (5), we wondered whether the observed anemia-induced downregulation of hepcidin could be conversely explained by iron deficiency. However, in both models of anemia, the hepatic iron level was found to be either unaltered in phlebotomized mice (not shown) or even increased in the case of PHZtreated mice. Treatment of animals with the hemolytic $\mathrm{PHZ}$ agent is indeed known to result in a large influx of iron to the liver following the massive hemocatheresis (12). We found a fourfold increase in total liver iron $(173 \pm 4 \mu \mathrm{g}$ iron/g wet liver tissue in PHZ-treated mice, $n=4$, vs. $47 \pm 5 \mu \mathrm{g}$ in control, $n=3 ; P<0.0001)$ and a threefold increase in serum iron $(6.309 \pm 272 \mu \mathrm{g}$ serum iron/ 1 in PHZ-treated mice, $n=4$, vs. $1.991 \pm 315 \mu \mathrm{g}$ in control, $n=3 ; P<0.0001)$. This observation led us to hypothesize that, apart from iron, another signal might regulate hepcidin gene expression, and hypoxia stimulus was suspected.

The negative effect of anemia on hepcidin gene expression is dominant over the positive effect of iron. To gain insight into the crosstalk in the regulation of hepcidin gene expression, upregulation by iron (5) and downregulation by anemia/hypoxia, mice experimentally iron loaded for 2 months using iron-dextran were treated for 3 days (four injections) with PHZ. As previously reported by Pigeon et al. (5), we showed that when injected into animals, iron dextran resulted in a dramatic increase in hepcidin gene expression (Figure 3). Interestingly, we observed that these iron-dextran-treated animals were still sensitive to the inhibitory effect of PHZ; i.e., the high levels of iron-induced hepcidin mRNA were reduced to levels similar to that of the control animals (Figure 3).

Hepcidin gene expression is tightly controlled by bypoxia. To investigate the effect of hypoxia on hepcidin gene expression, we first took advantage of the HepG2 and Hep3B human hepatoma cells that are widely used to study the response of liver gene expression to the hypoxic stress. These hepatoma cells were cultured either under normoxia ( $20 \%$ oxygen) or under hypoxic conditions $(10 \%, 2 \%$, and $0.1 \% \mathrm{O}_{2}$ ) for 24 hours. Expression of the human hepcidin gene was assessed by Northern blot analysis. As shown in Figure 4a, hepcidin gene expression was dramatically decreased in HepG2 cells cultured under hypoxia. This decrease in hepcidin gene expression was significant at $2 \% \mathrm{O}_{2}$, and the signal corresponding to the hepcidin mRNA was barely detectable at $0.1 \% \mathrm{O}_{2}$. The decrease in hepcidin gene expression was specific since under the same conditions of hypoxia expression of the vegf gene was upregulated, as expected (8). A similar specific decrease in hepcidin gene expression was also observed in Hep3B human hepatoma cells cultured under hypoxia (data not shown).

To check whether this result was also valid in vivo, hepcidin gene expression was studied in mice housed in hypobaric hypoxia chambers (simulated altitude of $5,500 \mathrm{~m}$ ) for $1,2,4$, and 12 days. As evidenced on the Northern blot seen in Figure 4b, hepcidin gene expression was strongly downregulated by 2- and 4-day hypoxic treatments of the mice (threefold decrease; $P<0.0001$ ). After 12 days of hypoxia, depending on the animal, hepcidin mRNA level either remained low (animals 4 and 8 ) or was similar to the normoxic values (animals 12 and 16). In Figure 4c, we showed by RT-PCR that the hepc1 transcripts followed the same variation under hypoxia. Since anemia results in tissue hypoxia, these results suggest that tissue oxygenation can constitute a major cue for hepcidin gene regulation. In addition to its role in mediating the iron absorption response to hypoxia, the question can be raised whether hepcidin could also be causally involved in the anemias associated with chronic inflammatory diseases.

Hepcidin gene expression is dramatically induced during acute inflammatory process. A mild normocytic anemia is known to occur in chronic inflammatory states. It is characterized by reduced plasma iron and storage of iron in the reticuloendothelial system. Such a mild ane- 


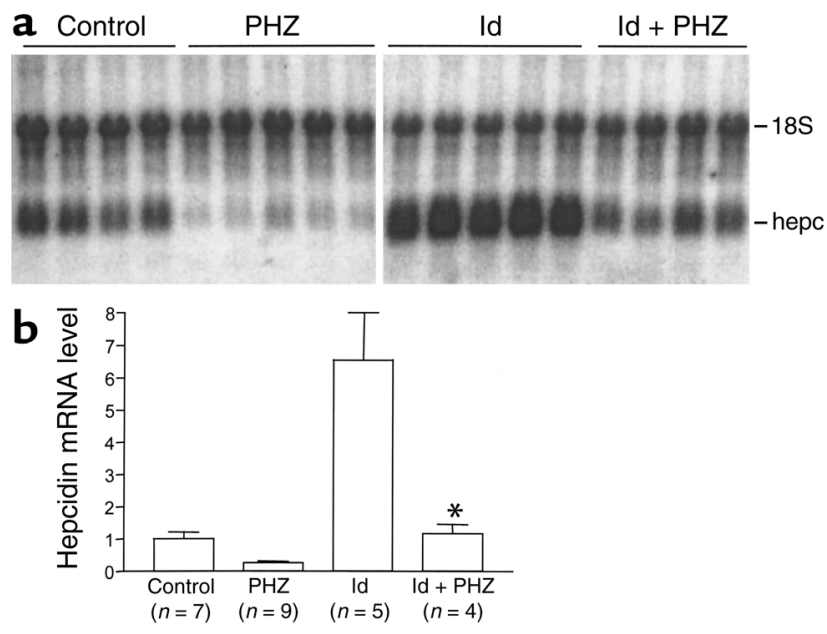

mia was induced in mice by chronic turpentine treatment consisting of weekly injections for 2 weeks (three injections) and sacrifice 4 weeks after the last injection. As determined in Figure 5a, this chronic treatment indeed resulted in anemia with significant reduction of key blood parameters, i.e., red cell mass, HGB, and HCT. Early after turpentine injection (at the 16th hour), a sixfold increase in hepcidin gene expression in the liver was detected $(P<0.0001$ as compared with saline-injected control; Figure 5, b and c). At this time, as previously reported (13), serum iron was decreased by twofold (data not shown). The increase in hepc1 transcripts as revealed by RT-PCR is shown in Figure $5 \mathrm{~d}$. The hepcidin gene expression was induced as soon as 6 hours after turpentine injection (twofold increase; $P<0.05$, not shown) and returned to normal values several days after the first injection.

The effect of turpentine injection for 16 hours was assessed in our previously described hepcidin-deficient

\section{Figure 3}

Effect of PHZ treatment in control mice and experimentally ironoverloaded animals. (a) Hepcidin mRNA content in liver of control mice, mice treated with $\mathrm{PHZ}$, mice experimentally iron overloaded, and mice iron overloaded and treated with $\mathrm{PHZ}$ (see Methods) as determined by Northern blot analysis. Twenty micrograms of total liver RNAs were electrophoresed, blotted, and hybridized with both hepcidin and 18S-labeled probes (as described in Methods). Id, iron dextran. (b) Relative changes in hepcidin. mRNA level (18S normalized, arbitrary units) as calculated using a STORM850 Phospholmager and ImageQuant 5.0. Results are expressed as mean \pm SD for $n$ animals, and statistical analysis was performed using the Student $t$ test (unpaired, two-tailed). ${ }^{*} P<0.001$ as compared with iron-overloaded mice.

mice and compared with that of wild-type mice of the same genetic background (6). As shown in Figure 6a, as expected, a twofold decrease in serum iron was measured in the wild-type mice injected for 16 hours with turpentine $(P<0.0001$, as compared with mice before turpentine injection). Very interestingly, this effect of turpentine-induced decrease of serum iron was not observed in hepcidin-deficient mice (Figure 6b).

\section{Discussion}

Anemia, bypoxia, and hepcidin. In this article we have demonstrated that experimentally induced anemia triggers a considerable decrease in hepcidin mRNA level in mice. We also demonstrated that hypoxia was able to reduce the level of hepcidin transcripts ex vivo in human HepG2 and Hep3B hepatoma cells and in vivo in mice housed in hypobaric hypoxia chambers. We hypothesized that the increase in intestinal absorption seen in anemia and hypoxia $(2,14)$ is due to the decrease in hep-

\section{Figure 4}

Hepcidin mRNA levels under ex vivo and in vivo hypoxic conditions. (a) Hepcidin mRNA content in HepG2 hepatoma cell lines as determined by Northern blot analysis. HepG 2 cells were untreated $\left(20 \% \mathrm{O}_{2}\right)$ or treated with 10,2 , or $0.1 \% \mathrm{O}_{2}$. Twenty micrograms of total liver RNAs were electrophoresed, blotted, and hybridized with both human hepcidin and 18S-labeled probes (as described in Methods). RNAs from HepG2 cells were also hybridized with a human VEGF cDNA probe as a control of the simulation of the vegf gene under hypoxia. (b) Hepcidin mRNA content in liver of mice housed in hypobaric hypoxic chambers for 1, 2, or 4 days, as determined by Northern blot analysis. Twenty micrograms of total liver RNAs were electrophoresed, blotted, and hybridized with both hepcidin and 185 -labeled probes (as described in Methods). Each lane represented different animals. (c) Level of specific hepc 1 transcripts in the liver as measured by RT-PCR (as described in Methods). Following PCR, the amplified products (171 bp for hepc 1 and 250 bp for $\beta$-actin) were separated by electrophoresis on $1.5 \%$ agarose gel.
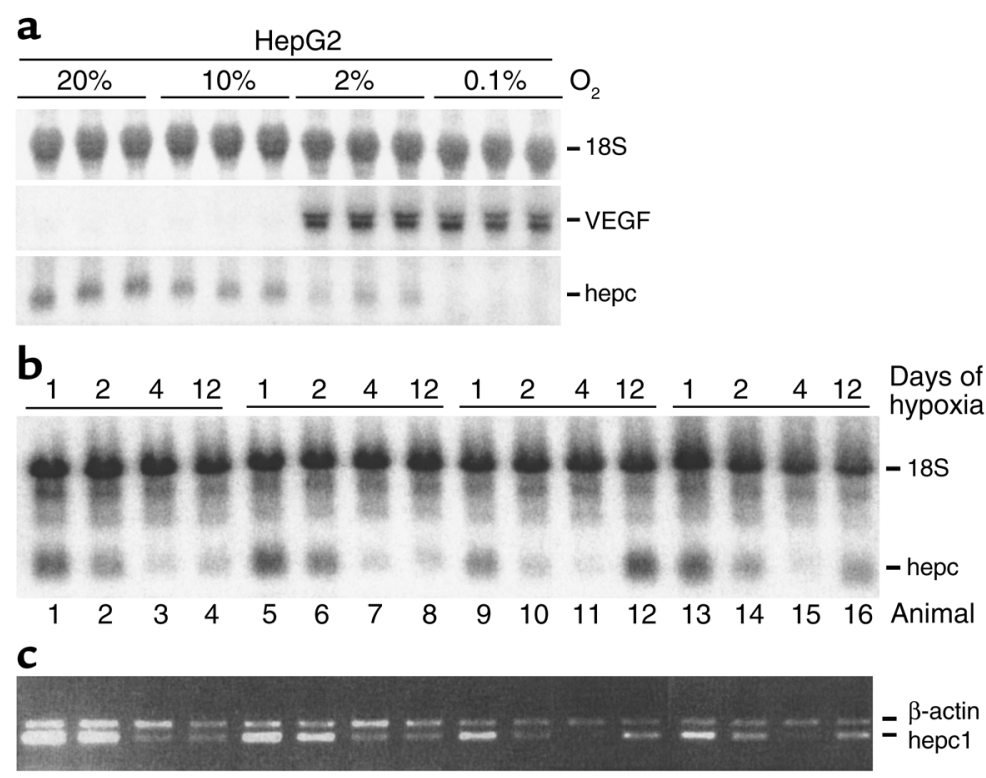


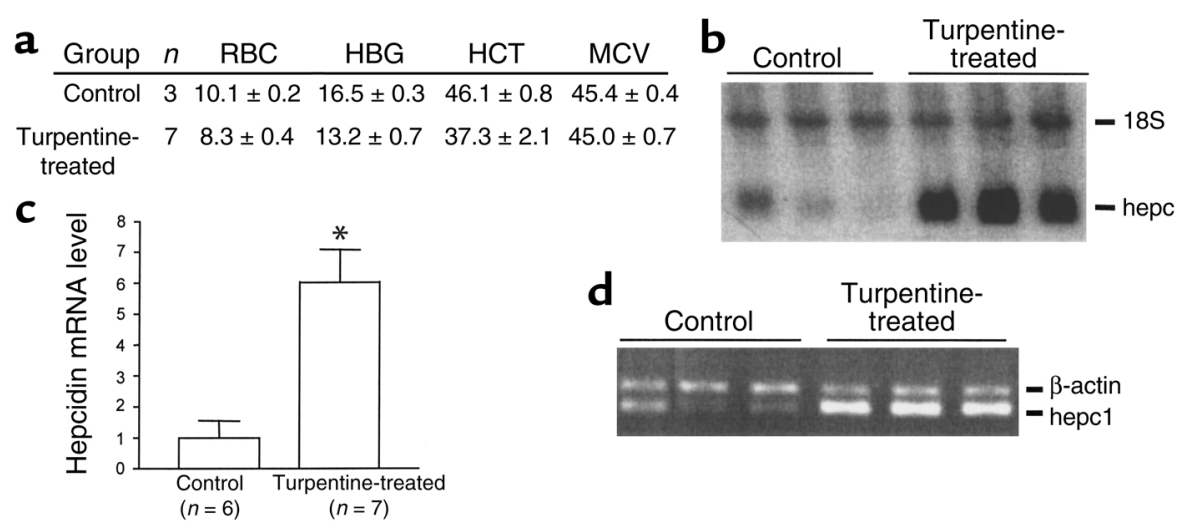

\section{Figure 5}

Hematological indices and hepcidin mRNA levels in turpentine-treated mice. (a) Hematological parameters in mice following chronic turpentine treatment: RBC $\left(10^{6} / \mathrm{ml}\right)$, HBG concentration $(\mathrm{g} / \mathrm{dl}), \mathrm{HCT}(\%)$, and $\mathrm{MCV}(\mathrm{fl})$. Results are expressed as mean $\pm \mathrm{SEM}$ for $n$ animals. (b) Hepcidin mRNA content in liver of control and 16-hour turpentine-treated mice as determined by Northern blot analysis. Twenty micrograms of total liver RNAs were electrophoresed, blotted, and hybridized with a 32P-labeled probe (as described in Methods) for hepcidin. Autoradiography of a typical experiment. (c) Relative changes in hepcidin mRNA level (18S normalized, arbitrary units) as calculated using a STORM850 Phospholmager and ImageQuant 5.0. Results are expressed as mean \pm SD for $n$ animals, and statistical analysis was performed using the Student $t$ test (unpaired, two-tailed). ${ }^{*} P<0.0001$. (d) Level of specific hepc1 transcripts in the liver as measured by RT-PCR (as described in Methods). Following PCR, the amplified products (171 bp for hepc1 and 250 bp for $\beta$-actin) were separated by electrophoresis on $1.5 \%$ agarose gel.

cidin gene expression. Raja et al. have reported that normal mice exposed to hypoxia for 3 days exhibit a two- to threefold increase in iron absorption (2). Interestingly, the authors also demonstrated that turpentinetreated mice exposed to 3 days of hypoxia failed to show this increase in iron absorption. This latter observation can be explained by the positive response of the hepcidin gene to inflammation counterbalancing the hypoxia-dependent downregulation of this gene.

A speculative scheme in the regulation of iron balance by hepcidin is proposed in Figure 7. When ane$\mathrm{mia} /$ hypoxia occurs, e.g., following severe bleeding or after PHZ treatment, erythropoietin expression increases, leading to a stimulation of the erythropoietic activity. In parallel, hepcidin gene expression is decreased, inducing a rapid mobilization of iron from reticuloendothelial cells to supply sufficient amounts of iron for the erythropoietic activity. Indeed, we demonstrated previously that a deficiency in hepcidin gene expression results in a dramatic decrease in iron stores in reticuloendothelial cells (6). At the moment, we cannot specify whether erythropoietin is involved in hepcidin downregulation or whether hepcidin and erythropoietin responses to hypoxia are independent. The second effect, probably acting in the longer term, is the programming of the crypt cells to increase their iron absorptive capacities as they mature into absorptive enterocytes. When normoxic conditions are restored, if iron is not deficient, hepcidin gene expression returns to normal. If iron is deficient, according to the positive role of iron on hepcidin gene expression (5), the hepcidin gene expression is maintained at a low rate, ensuring increased intestinal iron absorption until stores are repleted.

The inhibition of hepcidin in mice with PHZ-induced acute hemolysis is of particular significance to our understanding of the mechanisms of iron overload and hemosiderosis in patients with different types of chronic anemias with hemolysis and/ or dyserythropoiesis, namely thalassemias, intramedullary hemolytic anemia, sideroblastic anemia, and erythroblastopenia. Indeed, in irondextran PHZ-treated animals, the increase in liver and serum iron is unable to counteract anemia/hypoxia-induced downregulation of the hepcidin gene expression. In these animals, the inhibition of hepcidin gene expression, despite iron overload, can be considered as an inappropriate physiological response contributing to worsening iron overload, leading to hemosiderosis and its numerous pathological conditions. In patients with the aforementioned hematological disorders, similar mechanisms may play a major role in the long-term prognosis of these pathologies. The only available means to slow down hemosiderosis development in these patients lies in a regular pump-dependent delivery of iron chelators. Our data suggest that, provided that hepcidin indeed plays a similar role in humans and rodents, delivery of hepcidin itself, or of hepcidin agonists, could constitute in the future a rational preventive treatment under such circumstances, avoiding the inappropriate intestinal iron absorption.
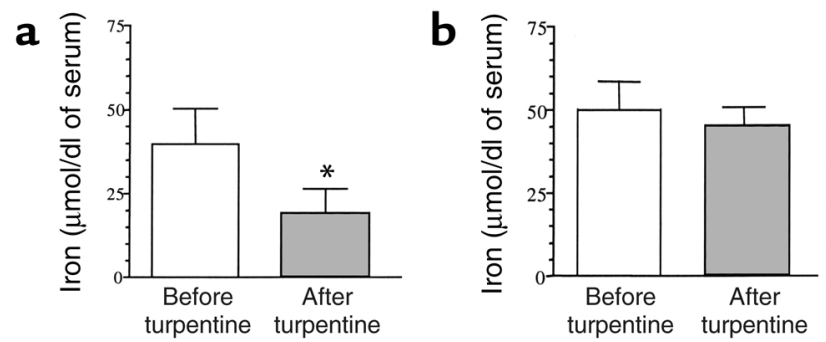

\section{Figure 6}

Effect of a single turpentine injection on serum iron content in wildtype and hepcidin-deficient mice. (a) Serum iron in wild-type mice before (white bar) and after (gray bar) a 16-hour turpentine injection. Results are expressed as mean \pm SEM for eight animals. Statistical analysis was performed using Student $t$ test (unpaired, two-tailed). ${ }^{*} P<0.0001$. (b) Serum iron in hepcidin-deficient mice before (white bar) and after (gray bar) a 16-hour turpentive injection. Results are expressed as mean \pm SEM for seven animals. 


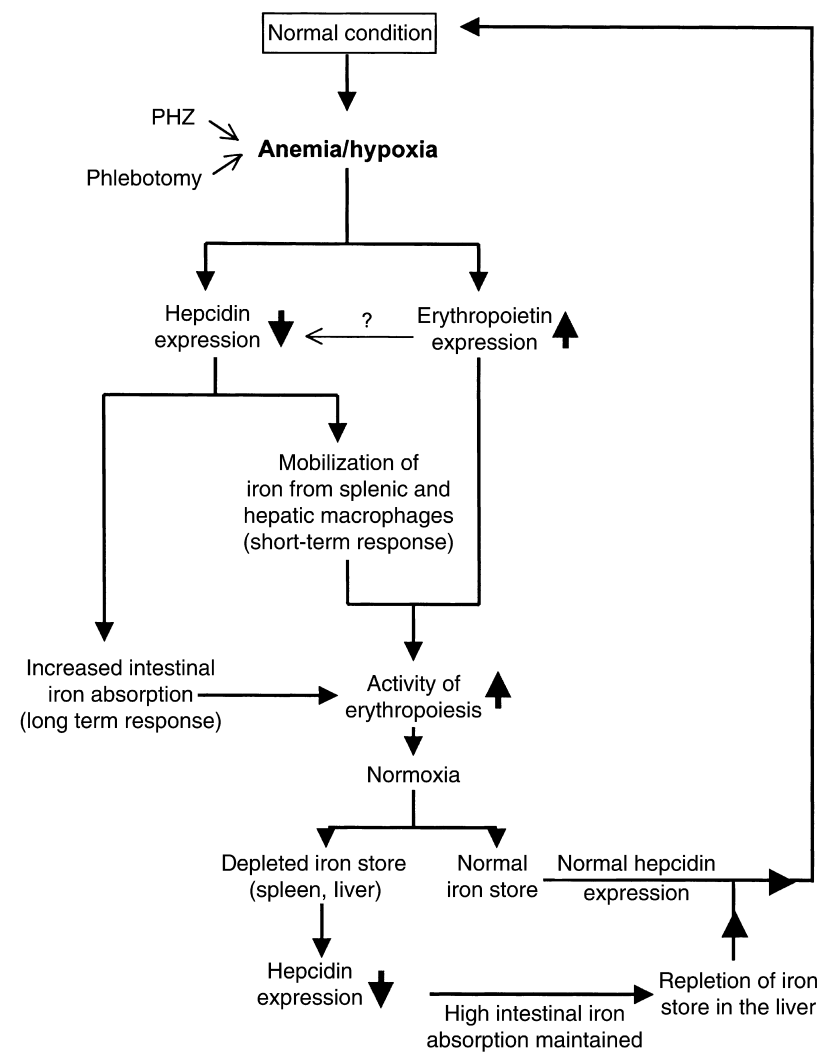

Figure 7

Scheme for suggested role of hepcidin for maintaining iron homeostasis following iron disturbance.

Interestingly, very recently Weinstein et al. (15) reported the first evidence of an effect of hepcidin in humans. In a cohort of type 1a glycogen-storage disease patients with chronic anemia, large hepatic adenomas were detected. The authors demonstrated that the unaffected liver tissue was producing a very low amount of hepcidin, consistent with the anemia, while, in contrast, the adenoma part was synthesizing an inappropriately high level of hepcidin. When the adenomas were removed, the anemia resolved spontaneously (15).

In addition to acting directly through tissue hypoxia, the question is raised whether anemia can also modulate iron absorption through an unknown cue related to erythropoietin-dependent erythropoiesis stimulation. In other words, does an erythropoietic regulator other than hepcidin exist, or can hepcidin be regulated according to the erythropoiesis need independently of hypoxia? In fact, the work by Peters and Coll (16) provides evidence that hypoxia enhances iron uptake by a mechanism that is independent of stimulated erythropoiesis. They have indeed demonstrated that exposure of splenectomized 89Sr-treated mice to hypoxia results in an increase in the total intestinal iron uptake, without any change in erythropoiesis (16). In agreement, these authors demonstrate that transgenic mice with hypoplastic anemia due to incomplete deficiency of erythropoietin have increased iron absorption, even in the absence of increased erythropoiesis (17). These results reinforce our hypothesis that hepcidin per se is a key component of the erythropoietic regulator of intestinal iron absorption.

Inflammation and hepcidin. Mice bearing sterile turpentine-induced abscesses and sacrificed 16 hours after the injection display a sixfold increase in hepcidin mRNA content in the liver. As for this turpentineinduced inflammation, we observed a dramatic increase in hepcidin gene expression 1 day after adenovirus-mediated inflammation (data not shown). These results, taken together with the previously reported upregulation of hepcidin gene expression by systemic inflammation induced by LPSs (5), strongly support a role for hepcidin as a positive acute-phase response peptide. The direct role of hepcidin in regulating iron metabolism during inflammatory states was brought up by studying the effect of turpentine in hepcidindeficient mice (Figure 6). Indeed, while acute inflammation produced by a single turpentine injection for 16 hours was leading to a twofold reduction of serum iron in wild-type mice, this hyposideremic effect of turpentine was completely blunted in hepcidin-deficient mice. This result clearly demonstrates that hepcidin is playing a key role in the dysregulation of iron homeostasis during inflammatory states.

Finally, in a recent hepatic transcriptome analysis, hepcidin mRNA was found to be increased during partial hepatectomy in mice (18). Further studies are now needed to define precisely the role of increased hepcidin in acute inflammation. Since hepcidin appears to be a defensin-like peptide, whose antimicrobial activity has been demonstrated, at least in vitro, we can hypothesize that the response of the hepcidin gene to stress and inflammation reflects the original role of this peptide $(3,4)$. Increased hepcidin secretion could then result in both rapid sequestration of iron by the reticuloendothelial cells, a constant phenomenon during inflammation (13), and inhibition of duodenal iron absorption, both contributing to decrease serum iron concentration (by $50 \%$ in turpentine-injected mice).

Interestingly, it has been reported that the inflammatory state, characterized by hyposideremia due to impaired release of iron by the reticuloendothelial system, may be modeled in vitro by incubating erythrophagocyting Kupffer cells with serum harvested from rats treated for 18 hours with turpentine (13). The authors indicate that they failed to identify the humoral agent responsible for this effect. Among the factors thought to be responsible for the manifestations of inflammation at the cellular level were IL-1, IFN- $\gamma$, and TNF, but none of these humoral agents appreciably influenced the course of iron release by Kupffer cells. We propose that hepcidin could be a bona fide agent, its upregulation in the liver leading to subsequent inhibition of iron release by reticuloendothelial cells, thus contributing to the anemia, as reported above. If true, the reverse situation, namely a decrease in hepcidin production, is expected to lead to an increased release of iron by the reticuloendothelial 
cells and increased intestinal iron absorption. This is, in fact, what we observed in hepcidin-deficient mice, where the reticuloendothelial cells from the spleen were shown to contain a very low amount of iron compared with control mice (6). Our data therefore suggest that the upregulation of the hepcidin gene by inflammation could play a major role in iron disorders and anemias observed in acute or chronic inflammatory diseases, i.e., infectious diseases, osteoarticular diseases such as rheumatoid polyarthritis, and even in malignancies, especially when associated with a severe inflammatory syndrome (Hodgkin disease, reticulosarcomas).

In conclusion, we have demonstrated that anemia and hypoxia on the one hand, and inflammation on the other, can regulate hepcidin gene expression in mice. The response of the hepcidin gene to hypoxia is also observed in human cells. Reduction of hepcidin production induced by tissue hypoxia may be an important cause of increased intestinal iron absorption that occurs even when iron stores are replenished. Consequently, if hepcidin plays a similar role in humans, administration of this peptide or of its agonists could be appropriate to avoid the severe iron overload complication in patients with chronic hemolytic and dyserythropoietic anemias. The hepcidin gene is an acute-phase responsive gene being overexpressed in response to inflammation in mice. This could explain both iron sequestration in reticuloendothelial cells and decreased intestinal iron absorption, hence decreased serum iron and anemia. Any means to counteract hepcidin overexpression in inflammatory diseases could therefore decrease their hematological consequences.

\section{Acknowledgments}

We thank Dominique Labie and Bernard Grandchamp for fruitful discussions and critical reading of the manuscript. We are grateful to Christine Ovejero for providing the adenovirus-treated mice and Gilles Pages for the kind gift of the human VEGF probe. We thank Michèle Caüzac, Jacqueline Bauchet, and Nicolas Sorhaindo for technical assistance, and Alan Strickland for the careful revision of the manuscript. This work was supported by INSERM and the Ministère de la Recherche. Gaël Nicolas was funded by the FRM, Fondation pour la Recherche Médicale and awarded by the Association Hémochromatose France.

1. Finch, C. 1994. Regulators of iron balance in humans. Blood. 84:1697-1702.

2. Raja, K.B., Duane, P., and Peters, T.J. 1990. Effects of turpentine-induced inflammation on the hypoxic stimulation of intestinal Fe3+ absorption in mice. Int. J. Exp. Pathol. 71:785-789.

3. Park, C.H., Valore, E.V., Waring, A.J., and Ganz, T. 2001. Hepcidin: a urinary antimicrobial peptide synthesized in the liver. J. Biol. Chem. 276:7806-7810.

4. Krause, A., et al. 2000. LEAP-1, a novel highly disulfide-bonded human peptide, exhibits antimicrobial activity. FEBS Lett. 480:147-150.

5. Pigeon, C., et al. 2001. A new mouse liver specific gene, encoding a protein homologous to human antimicrobial peptide hepcidin, is overexpressed during iron overload. J. Biol. Chem. 276:7811-7819.

6. Nicolas, G., et al. 2001. Lack of hepcidin gene expression and severe tissue iron overload in upstream stimulatory factor 2 (USF2) knockout mice. Proc. Natl. Acad. Sci. USA. 98:8780-8785.

7. Nicolas, G., et al. 2002. Severe iron deficiency anemia in transgenic mice expressing liver hepcidin. Proc. Natl. Acad. Sci. USA. 99:4596-4601.

8. Mazure, N.M., et al. 2002. Repression of alpha-fetoprotein gene expression under hypoxic conditions in human hepatoma cells: characterization of a negative hypoxia response element that mediates opposite effects of hypoxia inducible factor-1 and c-Myc. Cancer Res. 62:1158-1165.

9. Bigard, A.X., Sanchez, H., Birot, O., and Serrurier, B. 2000. Myosin heavy chain composition of skeletal muscles in young rats growing under hypobaric hypoxia conditions. J. Appl. Physiol. 88:479-486.

10. Milanini, J., Vinals, F., Pouyssegur, J., and Pages, G. 1998. p42/p44 MAP kinase module plays a key role in the transcriptional regulation of the vascular endothelial growth factor gene in fibroblasts. J. Biol. Chem. 273:18165-18172.

11. Torrance, J.D., and Bothwell, T.H. 1980. Tissue iron stores. Meth. Hematol. 1:90-115.

12. Ferrali, M., et al. 1997. Release of free, redox-active iron in the liver and DNA oxidative damage following phenylhydrazine intoxication. Biochem. Pharmacol. 53:1743-1751.

13. Kondo, H., Saito, K., Grasso, J.P., and Aisen, P. 1988. Iron metabolism in the erythrophagocytosing Kupffer cell. Hepatology. 8:32-38.

14. O'Riordan, D.K., et al. 1995. Cellular mechanisms underlying the increased duodenal iron absorption in rats in response to phenylhydrazine-induced haemolytic anaemia. Eur. J. Clin. Invest. 25:722-727.

15. Weinstein, D.A., et al. 2002. Inappropriate expression of hepcidin is associated with iron refractory anemia: implications for the anemia of chronic disease. Blood. In press.

16. Raja, K.B., Simpson, R.J., Pippard, M.J., and Peters, T.J. 1988. In vivo studies on the relationship between intestinal iron $(\mathrm{Fe} 3+)$ absorption, hypoxia and erythropoiesis in the mouse. Br. J. Haematol. 68:373-378.

17. Raja, K.B., et al. 1997. Iron metabolism in transgenic mice with hypoplastic anaemia due to incomplete deficiency of erythropoietin. Br. J. Haematol. 96:248-253.

18. Kelley-Loughnane, N., Sabla, G.E., Ley-Ebert, C., Aronow, B.J., and Bezerra, J.A. 2002. Independent and overlapping transcriptional activation during liver development and regeneration in mice. Hepatology. 35:525-534. 